\title{
Saccharopolyspora gregorii and Saccharopolyspora hordei: Two New Actinomycete Species from Fodder
}

\author{
By M. GOODFELLOW,${ }^{*}$ J. LACEY, ${ }^{2}$ M. ATHALYE,${ }^{1} \dagger$ \\ T. M. EMBLEY ${ }^{3}$ AND T. BOWEN ${ }^{3}$ \\ ${ }^{1}$ Department of Microbiology, The Medical School, Framlington Place, Newcastle upon Tyne \\ NE2 $4 H H, U K$ \\ ${ }^{2}$ AFRC Institute of Arable Crops Research, Rothamsted Experimental Station, Harpenden, \\ Herts AL5 2JQ, UK \\ ${ }^{3}$ Department of Paramedical Sciences, North East London Polytechnic, London E15 4LZ, UK
}

(Received 20 February 1989; revised 25 April 1989; accepted 10 May 1989)

\begin{abstract}
Spore-forming actinomycetes from fodder were compared with marker strains of the genera Amycolata, Amycolatopsis, Faenia, Saccharopolyspora and Saccharothrix. Data from 120 unit characters were examined using the pattern and simple matching coefficients, and clustering was achieved using the unweighted pair group method with arithmetic averages algorithm. Most of the fodder isolates were recovered in two major clusters which were equated with taxospecies. Representatives of the numerically circumscribed species contained mesodiaminopimelic acid, arabinose and galactose, were rich in iso- and anteiso- fatty acids, exhibited tetrahydrogenated menaquinones with nine isoprene units as the predominant isoprenologue and gave similar polar lipid patterns. The chemical, morphological and numerical phenetic data indicated that the organisms belong to the genus Saccharopolyspora. It is proposed that the two novel taxa be given species status within the genus Saccharopolyspora as Saccharopolyspora gregorii sp. nov. and Saccharopolyspora hordei sp. nov.
\end{abstract}

\section{INTRODUCTION}

Actinomycetes are common in fodder (Lacey, 1988), where they grow rapidly on heating and produce degradative enzymes that enable utilization of the substrate. Members of some taxa cause extrinsic allergic alveolitis (hypersensitivity pneumonitis) resulting from inhalation of large numbers of spores released when mouldy substrates are disturbed (Lacey, 1981). Species such as Faenia rectivirgula, Saccharomonospora viridis, Streptomyces albidoflavus and Streptomyces albus are found in a wide range of substrates but other taxa, notably Nocardiopsis dassonvillei, Saccharopolyspora hirsuta, Streptomyces thermoviolaceus and Thermomonospora chromogena, seem to be favoured by particular substrates (Lacey, 1988). Fodder isolates whose taxonomic status is uncertain include strains initially assigned to the genus Actinomadura (Lacey et al., 1978) but subsequently shown to form three numerically circumscribed taxa, clusters A, B and C (Athalye, 1981), that had little in common with phena corresponding to established species of Actinomadura (Goodfellow et al., 1979) or Streptomyces (Williams et al., 1983).

Chemical markers are now widely employed in the circumscription of actinomycete genera (see Williams et al., 1989), although numerical taxonomy still provides an effective way of assigning numerous poorly classified organisms to taxospecies (see Goodfellow \& Dickinson, 1985). Actinomycetes which contain meso-diaminopimelic acid, arabinose and galactose (wall chemotype IV sensu Lechevalier \& Lechevalier, 1970), but lack mycolic acids, form the

† Present address: Beecham Pharmaceuticals Research Division, Chemotherapeutic Research Centre, Brockham Park, Betchworth, Surrey RH3 7AJ, UK. 
micropolysporas (Goodfellow \& Cross, 1984). This suprageneric group accommodates not only the established genera Actinopolyspora, Amycolata, Amycolatopsis, Faenia, Pseudonocardia, Saccharomonospora and Saccharopolyspora but also fodder isolates assigned to clusters A and B (Goodfellow et al., 1979). Members of these genera have DNA that is especially rich in guanine (G) plus cytosine (C) and are characterized by a combination of chemical and morphological properties (Goodfellow \& Minnikin, 1984; Lechevalier et al., 1986; Embley et al., 1988).

The present study was designed to determine the taxonomic status and affinities of strains belonging to clusters $\mathrm{A}$ and $\mathrm{B}$ by comparing them, using chemical and numerical taxonomic criteria, with marker strains of established wall chemotype IV taxa lacking mycolic acids. The test strains were isolated from cereal grains, hay and sugar-cane bagasse using half-strength nutrient agar supplemented with cycloheximide and a wind tunnel/Andersen sampler method (Gregory \& Lacey, 1963; Lacey, 1971). Isolates assigned to cluster A were most frequently isolated from barley grain and less often from hay and sugar cane bagasse. They were present, together with Faenia rectivirgula, in $15 \%$ of freshly harvested grain samples but not in grass used to seal moist barley silos. However, after storage, they occurred in $33 \%$ of grass and straw samples and in $57 \%$ and $32 \%$ of grain samples in two seasons (Lacey, 1971). The cluster B isolates chiefly came from hay but they occurred in few samples and were rarely numerous.

\section{METHODS}

Strains. The 115 test organisms included representative fodder isolates and reference strains from private and service culture collections (Table 1). Where possible, type strains were included; duplicated cultures of seven strains were used as internal checks on experimental test error. The organisms were maintained on glucose/yeast extract agar (GYEA; Gordon \& Mihm, 1962) and as suspensions of spores or mycelial fragments in glycerol $(20 \%$, $\mathrm{v} / \mathrm{v})$ stored at $-20^{\circ} \mathrm{C}$.

Data acquisition. Each strain was examined for 120 unit characters (Table 2) using procedures described previously (Athalye $e t$ al., 1985). Unless otherwise stated, tests were incubated at $30^{\circ} \mathrm{C}$ and results recorded weekly for up to $28 \mathrm{~d}$ apart from those with Faenia rectivirgula, which were incubated at $45^{\circ} \mathrm{C}$ for $14 \mathrm{~d}$. Tests were repeated only when ambiguous or clearly unexpected results were obtained. Where possible, they were done in Replidishes (Sterilin) (Table 2) which were inoculated, with one exception, from 10- to 14-d-old GYEA master Replidishes using a multipoint inoculation procedure (Sneath \& Stevens, 1967). The antibiotic sensitivity tests were inoculated using 7-d-old glucose/yeast extract broth cultures. Inocula for the remaining tests were taken from freshly thawed glycerol suspensions. Data for computation were derived from final test readings apart from the antibiotic sensitivity tests, which were recorded after $5 \mathrm{~d}$; that is, when good growth was apparent on the positive controls.

Coding of data. Nearly all of the characters existed in one of two mutually exclusive states that were scored as plus (1) or minus (0). Qualitative multistate characters, such as pigmentation and colony elevation, were recorded as several independent features scored plus (1) for the character state shown and minus (0) for all other alternatives. The final data matrix contained information on 122 strains and 120 unit characters.

Computation. Data were examined using the CLASP package of programs (written by G. J. S. Ross, F. B. Laukner and D. Hawkins, Rothamsted Experimental Station, Harpenden, UK) on an ICL 470/472 computer, using the simple matching coefficient $\left(S_{\mathrm{SM}}\right.$; Sokal \& Michener, 1958), which includes both positive and negative similarities, and the pattern coefficient $\left(D_{\mathrm{p}}\right)$, which allows for differences in growth rates, periods of incubation and similar factors that can distort similarity values (Sneath, 1968). Clustering was achieved using the unweighted pair group method with arithmetic averages (UPGMA) algorithm (Sneath \& Sokal, 1973).

Strains and growth conditions for chemosystematic analyses. Biomass for the fatty acid, isoprenoid quinone, polar lipid and wall amino acid analyses was prepared by growing strains representing the Saccharopolyspora species (Table 4) in shake flasks of nutrient broth (Oxoid CM3) for 7 to $10 \mathrm{~d}$ at $30^{\circ} \mathrm{C}$. Cultures were checked for purity at maximum growth, killed with formalin $(1 \%, \mathrm{v} / \mathrm{v})$, harvested by centrifugation, washed with distilled water and freeze-dried. Three representatives of cluster C, strains A258, A259 and A261, grown under the same conditions were used for diaminopimelic acid analyses. For the DNA base composition studies, strains A65 (cluster A) and A85 (cluster B) were grown in nutrient broth to early exponential phase then harvested by centrifugation.

DNA base composition studies. Harvested biomass was washed with TES buffer $(0.05 \mathrm{M}$-Tris, $0.05 \mathrm{M}-\mathrm{NaCl}$, $0.005 \mathrm{M}$-EDTA; $\mathrm{pH} 8.0$ ). The cells were then resuspended in $20 \mathrm{ml}$ TES buffer containing sodium dodecyl sulphate $(0.5 \%, \mathrm{v} / \mathrm{v})$ and proteinase $\mathrm{K}$ (Sigma $500 \mu \mathrm{g} \mathrm{ml}^{-1}$ ) and disrupted by passage through a pre-cooled French pressure cell at 8000 p.s.i. ( $55 \mathrm{MPa}$ ). The DNA samples were then purified further and the thermal denaturation method used to determine the $\mathrm{G}+\mathrm{C}$ content of DNA extracted from the test organisms (Marmur, 1961). Determinations were performed in $0.1 \times \mathrm{SSC}(1 \times \mathrm{SSC}$ is $0.15 \mathrm{M}-\mathrm{NaCl}$ plus $0.015 \mathrm{M}$-trisodium citrate). Escherichia coli DNA (Sigma) with a G+C content of $51 \mathrm{~mol} \%$ was used as an internal standard. 
Extraction and analysis of fatty acid methyl esters (FAMEs). Dried biomass $(30 \mathrm{mg})$ was degraded by acid methanolysis (Minnikin et al., 1980) and analytical and preparative TLC of the methanolysates was carried out as described previously (O'Donnell et al., 1982a). Capillary GC of the FAMEs was performed according to Embley et al. (1987). The retention times and relative proportions of each FAME were determined using a Shimadzu CR3A computing integrator. The identity of individual esters was established by comparison of the retention times with those of fatty acids isolated from Saccharopolyspora hirsuta ATCC 27875 (Embley et al., 1987).

Extraction and analysis of polar lipids and isoprenoid quinones. The small-scale procedure of Minnikin et al. (1984) was used to extract these lipids from biomass samples (about $150 \mathrm{mg}$ ). Purified isoprenoid quinones were analysed by mass spectrometry and quantified by reverse-phase HPLC (Collins, 1985). Polar lipid patterns were obtained using published procedures (Minnikin et al., 1984). Choline-positive spots were detected on thin-layer plates using Draggendorf's reagent (Wagner et al., 1961).

Cell wall amino acid analysis. Cell walls and heptafluorobutyrate isobutyl ester derivatives of amino acids were prepared as described by O'Donnell et al. (1982b) and analysed using capillary GC (Embley et al., 1984). The isomeric forms of diaminopimelic acids were determined by chromatography of cell wall hydrolysates on thinlayer sheets (Eastman Kodak 13255) after Staneck \& Roberts (1974). Sugars in whole-organism hydrolysates were analysed as their alditol acetates on a Silar $10 \mathrm{C}$ (Altech Associates) capillary column using the simplified method of Englyst \& Cummings (1984).

Morphological and growth studies. Additional morphological data on isolates were obtained from cultures growing on half-strength nutrient agar at 25 or $40^{\circ} \mathrm{C}$. Colonies were examined in situ on the agar surface using a compound microscope with $\times 20$ and $\times 40$ objectives.

Spores, collected by touching collodion-coated copper grids onto sporulating cultures, were examined without further treatment using a Siemens Eliniskop 1A transmission electron microscope.

Growth temperature ranges were determined by incubating cultures on half-strength nutrient agar at different temperatures between 10 and $60^{\circ} \mathrm{C}$, at $5^{\circ} \mathrm{C}$ intervals, and recording growth up to 3 weeks.

\section{RESULTS}

\section{Numerical classification}

The probability $(p)$ of an erroneous test result, as estimated from results with the six duplicated strains (Table 1), averaged $4.1 \%$, equal to an observed $S_{\mathrm{SM}}$ between duplicated cultures of just under $92 \%$. Cluster composition was not greatly affected by the statistical tests.

The test strains were assigned to six major ( 5 to 38 strains), six minor ( 2 to 3 strains) and ten single-member clusters defined at or above the 0.22 level in the $D_{\mathrm{P}}$, UPGMA analysis (Table 1 , Fig. 1). Most clusters were distinct and homogeneous and were named, where possible, after the type or authentic strains they contained. Clusters 1 to 6 , all of which encompassed isolates from fodder, showed a close affinity with the type strain of Saccharopolyspora hirsuta. Cluster 7 also contained fodder isolates together with strains received as Actinomadura madurae and Nocardiopsis dassonvillei (Table 1). The three strains received as Actinomadura species (cluster 12) were sharply distinguished from all of the other numerically defined clusters.

Cluster 1 accommodated 14 of the 26 cluster B strains. The remaining organisms provisionally assigned to cluster B were either recovered as single-member clusters or formed minor phena 2, 3,5 and 6 . In contrast, all but one of the 38 cluster A strains constituted cluster 4 ; the exception, strain A700, formed a single-member cluster. Similarly, clusters 7 and 9 encompassed the cluster $\mathrm{C}$ isolates and Saccharothrix aerocolonigenes, respectively. Amycolatopsis orientalis strains formed clusters 8 and 10, apart from $A$. orientalis N883, which was recovered as a distinct singlemember cluster. The organisms labelled Actinomadura that formed cluster 12 had properties typical of the genus Rhodococcus (Goodfellow, 1986). The affinities of Amycolata autotrophica A52 and other strains forming single-member clusters can be seen in Fig. 1.

Cluster composition was not greatly affected when the $S_{\mathrm{SM}}$ coefficient was employed, although some of the phena were arranged differently. The clusters were defined at or above the $77 \%$ similarity ( $S$-) level. Major clusters 1 (Saccharopolyspora gregorii) and 7 (cluster C) were recovered in their entirety, as were minor clusters 3 (cluster B), 11 (Faenia rectivirgula) and 12 (Rhodococcus sp.). Similarly, Amycolata autotrophica A52, Amycolatopsis orientalis N883, Saccharopolyspora hirsuta N745 and fodder isolates A49, A61, A68 and A108 again formed single-member clusters. Cluster 4 (Saccharopolyspora hordei) in the $S_{\mathrm{SM}}$, UPGMA analysis 
Table 1. Designations and sources of strains assigned to clusters in the $D_{P}, U P G M A$ analysis

Cluster

1

3

4

5

6

7

N833-N836, N838, N839, N849*, N850, N851, N852, N853

N837, N848, N854

Laboratory number

A44-A46, A79

A85a ${ }^{a}$ N259a

A62, A66, N252

A53, A60, A81, A86, A89, N258

A71, N260

A213, A214

A55, A58, A65, A67, A70, A83, A84, A105, A215, A216 ${ }^{b}, 7^{b}$, A218, A219, A220, A221, N269

A54, A63, A64, A87, A91, A266, A267

A47, A56, A77c, A104c, A88

$\mathrm{A} 51^{d}, \mathrm{~N} 268^{d}, \mathrm{~A} 48, \mathrm{~A} 59$

$\mathrm{A} 78, \mathrm{~A} 158, \mathrm{~A} 159^{e}, \mathrm{~A} 238^{e}, \mathrm{~A} 160^{\circ}$ A252 $f$

A72 ${ }^{g}, \mathrm{~N} 261^{g}$

A57, A75, A90

A106, A107, A217, A256, A258, A259, A261, A262, A263

A254, A255, A257, A260, A264

A110

A120, A121

N855, N880, N881, N857, N858, N875, N876, N878

N859-N861, N863*, N879

A162, A163, A164

A103, A135, A136
Received as

Cluster B

Cluster B

Cluster A

Cluster B

Cluster B

Cluster C

Actinomadura madurae

Nocardiopsis dassonvillei

Amycolatopsis orientalis

Saccharothrix aerocolonigenes

Amycolatopsis orientalis

Faenia rectivirgula

Actinomadura spp.
Sourceł

JL A1248, JL A640, JL A336, JL A1247

JL A333, CUB 355 (JL A333); hay

JL A66, JL A694, straw; CUB 130, soil

JL A670, JL A671, JL A637, JL A320, A674, CUB 253 (JL A321); barley

JL A548, CUB 359, (JL A409); barley

JL A1558, JL A1559; barley

JL A428, JL A471, JL A868, JL A491, JL A305, JL A581, JL A470, JL A474, JL A1560, JL A1561 ${ }^{b}$, JL A1563, JL A1560, JL A1565, JL A1566, CUB 437 (JL A683); barley

JL A735, JL A1276, JL A826, JL A1016, JL A620, JL A1234, JL A1549; hay

JL A676, JL A894, JL A232c, JL A824; sugar cane bagasse

JL A337, CUB 435 (JL A337), oats; JL A678, grain; JL A795, dust

JL A789, JL A1618, JL A1619, NCPF 1184 (JL A1619), JL A1620, NCPF 1185 (JL A 1620)

JL A410, CUB 360 (JL A410); barley

JL A879, coffee beans; JL A673, barley; JL A272, straw

JL A670, JL A888, JL A1562, JL A317, JL A436, JL A455, JL A506, JL A544, JL A624; barley

JL A204, bagasse; JL Ȧ282, JL A331, hay; JL A488, air; JL A1041, maize

CDC N248; sputum

IMRU 1250, IMET 9653; soil

IMRU 1148, IMRU 1627, IMRU 1628 (NRRL B-2452), IMRU 1389, IMRU 1390, IMRU 1131, IMRU 1409, clinical specimens; IMRU 1592, soil

IMRU 550, IMRU 938, IMRU 1106 , IMRU 1566 (NRRL B-1126T), IMRU 1593 (CDC W-2104), IMRU 1663, IMRU 1546 (NRRL B-3274), IMRU 1594 (CDC W-2105), IMRU 1599 (CDC W-2158), IMRU 1667, IMRU 1668; soil IMRU 1589 (CDC W-1894), spinal fluid; IMRU 1432, IMRU 1700

IMRU 1600 (NRRL B-3891), IMRU 1632, IMRU 1633, ISP 5040 (ATCC 19795), IMRU 1601; soil

JL A1622 (INMI 682), JL A1623 (INMI 1325), JL A1624 (INMI 9553)

RIA 452, CCM 5509, CCM 5510 
Table 1 (continued)

\begin{tabular}{|c|c|c|c|}
\hline Cluster & Laboratory number & Received as & Source $\ddagger$ \\
\hline \multirow[t]{6}{*}{ SMC $†$} & A $52^{*}$ & $\begin{array}{l}\text { Amycolata } \\
\text { autotrophica }\end{array}$ & JL A1269 (IMRU 1595, ATCC 19727) \\
\hline & N883 & $\begin{array}{l}\text { Amycolatopsis } \\
\text { orientalis }\end{array}$ & IMRU 1644 (CDC W-2648); spinal fluid \\
\hline & N745* & & JL A1143 (ATCC 27875); sugar cane bagasse \\
\hline & $\mathrm{A} 61, \mathrm{~A} 68, \mathrm{~A} 265$ & B & $\begin{array}{l}\text { JL A893, bagasse; JL A302, straw; } \\
\text { JL A1046, hay }\end{array}$ \\
\hline & 700 & & Laboratory isolate \\
\hline & v25l & $\begin{array}{l}\text { Fodder } \\
\text { isolates }\end{array}$ & $\begin{array}{l}\text { JL A286, barley; JL A1273, hay; N251, } \\
\text { CUB } 123\end{array}$ \\
\hline \multicolumn{4}{|c|}{$\begin{array}{l}\text { * Type strain. } \\
\text { a-g Duplicated cultures. } \\
\text { † SMC, single-member clusters. } \\
\text { † Sources: ATCC, American Type Culture Collection, Rockville, Maryland, USA; CCM, Czechoslovak } \\
\text { Collection of Micro-organisms, J. E. Purkyne University, Brno, Czechoslovakia; CDC, Center for Disease } \\
\text { Control, Atlanta, Georgia, USA; CUB, Dr T. Cross, School of Biomedical Sciences, University of Bradford, } \\
\text { Bradford, UK; IMET, Dr H. Prauser, Institute of Microbiology and Experimental Therapy, Jena, GDR; INMI, } \\
\text { Institute of Microbiology, The USSR Academy of Sciences, Moscow, USSR; INRU, Institute of Microbiology, } \\
\text { Rutgers University, New Brunswick, New Jersey, USA; NCPF, National Collection of Pathogenic Fungi, } \\
\text { Central Public Health Laboratory, Colindale, London, UK; JL, Dr J. Lacey, Rothamsted Experimental Station, } \\
\text { Harpenden, UK; NRRL, Northern Regional Research Laboratory, Agricultural Research Division, Peoria, } \\
\text { USA; RIA, The USSR Research Institute for Antibiotics, Moscow, USSR; RV, Professor R. Vanbreuseghem, } \\
\text { Institute of Tropical Medicine, Antwerp, Belgium. }\end{array}$} \\
\hline
\end{tabular}

accommodated 37 strains which were divided between two adjoining subclusters at the $72 \%$ $S$-level. Subcluster 4A contained 34 isolates, including strains A700 and N251 which had previously formed single-member clusters, and subcluster 4B contained strains A51, A53 and A78. The Saccharothrix aerocolonigenes strains were also recovered in two subclusters. Subcluster 9A encompassed 12 strains and was sharply separated from subcluster 9B, which contained strains N852 and N853. In contrast, phena 2 (cluster B) and 5 (cluster B) formed a single cluster that also contained strain A78 from phenon 4 (cluster A) and strain A90 from phenon 6 (cluster B). Similarly, the Amycolatopsis orientalis strains of clusters 8 and 10 formed a single cluster, apart from strain N883, which was again recovered as a single-member cluster. Phenon 6 (cluster B) contained three organisms, having gained strain A265, previously a singlemember cluster.

The properties of the multimembered clusters defined in the $D_{\mathrm{P}}$, UPGMA analysis are given in Table 2.

Species of Saccharopolyspora can be distinguished using a combination of biochemical, morphological, nutritional and physiological features (Table 3).

\section{Morphology}

Isolates classified in clusters $1,2,3$ and 5 were similar in appearance. Colonies were pale yellowish to warm buff and grew over a temperature range of $10-20$ to $35-40{ }^{\circ} \mathrm{C}$, mostly at 15 to $35^{\circ} \mathrm{C}$. There was usually extensive fragmentation of the substrate mycelium into rod-shaped and coccoid elements. White aerial mycelium was formed sparsely, if at all, with most produced by cluster 1 isolates, especially at $20^{\circ} \mathrm{C}$. Little was produced below 15 or above $25^{\circ} \mathrm{C}$. Electron microscopy showed mostly vegetative cells. Spores, with a smooth sheath, were only evident in strain A86, which was also atypical in having aerial mycelium with short side branches.

Cluster 4 isolates grew at higher temperatures than those of clusters $1,2,3$ and 5 . The temperature range for growth was $20-30$ up to $60^{\circ} \mathrm{C}$. Colonies were colourless to light or warm buff. The substrate mycelium showed some fragmentation into coccoid elements. When 


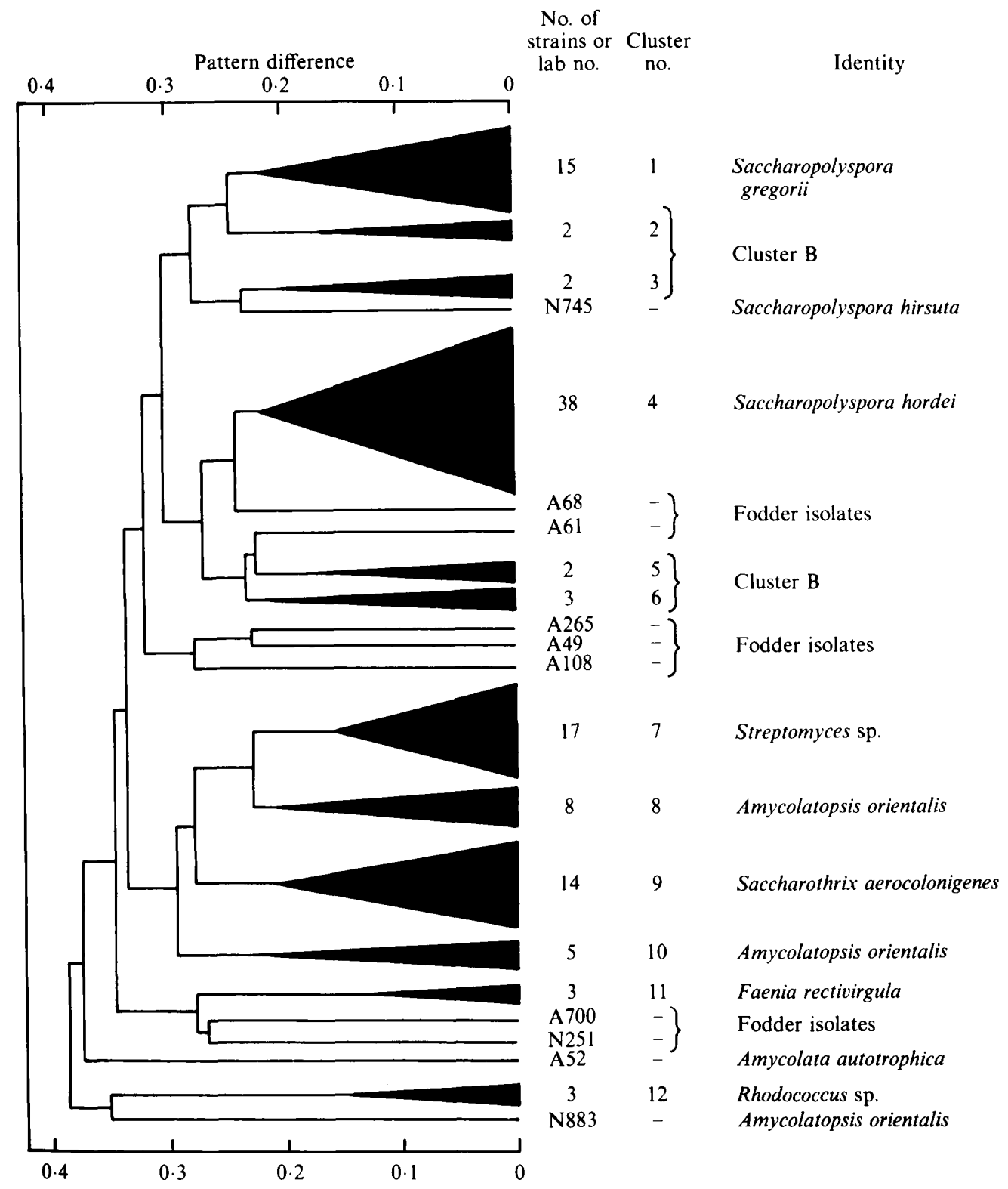

Fig. 1. Simplified dendrogram showing relationships amongst clusters based on the $D_{\mathrm{P}}$, UPGMA analysis.

produced, aerial mycelium was sparse, white and carried short, flexuous or curved spore chains (Fig. 2). Electron microscopy revealed the spores to be covered with a smooth or slightly warty sheath (Fig. 3) and adjacent spores were often separated by an empty segment of hypha, as observed previously in Saccharopolyspora hirsuta (Lacey \& Goodfellow, 1975). Isolated spores often had an annulus of sheath material evident at each end (Fig. 4). Strain A65 was atypical in growing only between 20 and $30^{\circ} \mathrm{C}$ and in having short, straight branches on aerial hyphae.

Two of the three cluster 6 isolates grew over the range 20 to $60^{\circ} \mathrm{C}$ while strain A57 grew only between 15 and $35^{\circ} \mathrm{C}$. Strains A75 and A90 resembled cluster 4 isolates while strain A57 colonies were colourless with little fragmentation or evidence of spores. 
Table 2. Distribution of positive characters to major and minor clusters defined in the $D_{P}, U P G M A$ analysis

Values are numbers of strains with positive character states

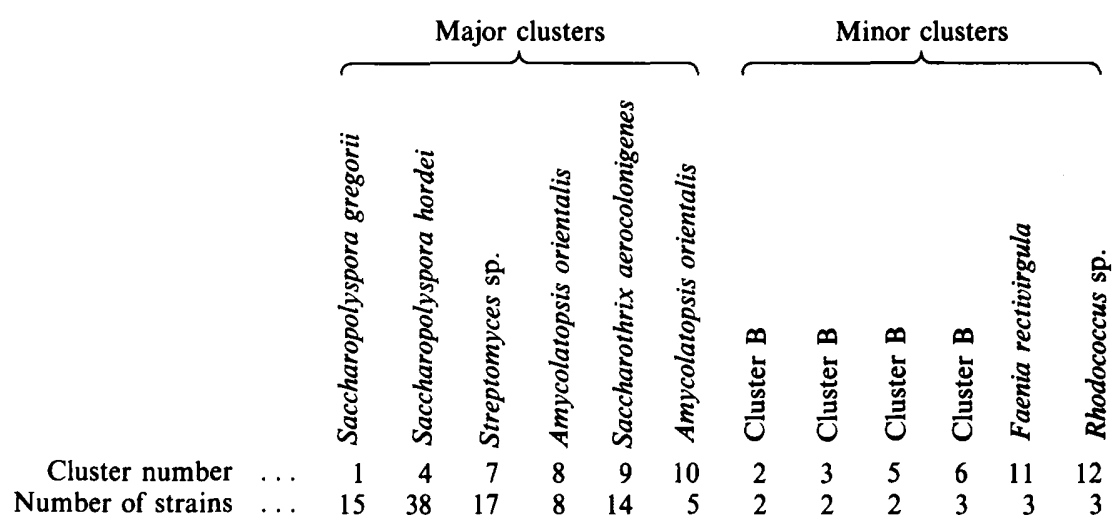

Biochemical tests:

Allantoin hydrolysed

Melanin produced

Nitrate reduced

Urea hydrolysed

Colony properties:

Aerial mycelium

Sparse

Moderate

Abundant

Colonies

Brown

Pink/orange

White/cream/yellow

Colony elevation

Convex

Crateriform

Irregular

Colony margin

Entire

Filamentous

Highly filamentous

Diffusible pigment

\section{Brown}

Yellow/orange

Degradation of:

Aesculin
Arbutin
Casein
DNA
Elastin
Gelatin
Guanine
Hypoxanthine
RNA
Starch
Testosterone
Tween 20
Tween 80
L-Tyrosine
Xanthine
Xylan

$$
\begin{array}{rrrrrrrrrrrr}
0 & 1 & 12 & 6 & 10 & 5 & 1 & 0 & 0 & 0 & 0 & 7 \\
8 & 3 & 3 & 0 & 5 & 0 & 0 & 1 & 1 & 1 & 0 & 2 \\
4 & 11 & 14 & 5 & 10 & 5 & 1 & 2 & 1 & 1 & 1 & 3 \\
1 & 4 & 14 & 7 & 11 & 5 & 1 & 1 & 0 & 1 & 0 & 2
\end{array}
$$$$
\begin{array}{llllllllllll}
1 & 0 & 2 & 1 & 3 & 0 & 0 & 0 & 0 & 1 & 3 & 0 \\
0 & 0 & 6 & 5 & 1 & 0 & 0 & 0 & 0 & 1 & 0 & 0 \\
0 & 0 & 3 & 2 & 1 & 1 & 0 & 0 & 0 & 0 & 0 & 0
\end{array}
$$$$
\begin{array}{llllllllllll}
0 & 0 & 0 & 0 & 1 & 0 & 0 & 0 & 0 & 0 & 0 & 0 \\
0 & 0 & 0 & 0 & 0 & 0 & 0 & 0 & 0 & 0 & 0 & 3
\end{array}
$$$$
\begin{array}{llllllllllll}
15 & 37 & 17 & 8 & 14 & 5 & 2 & 2 & 2 & 3 & 3 & 0
\end{array}
$$$$
\begin{array}{llllllllllll}
4 & 17 & 1 & 2 & 1 & 0 & 0 & 0 & 2 & 1 & 0 & 1
\end{array}
$$$$
\begin{array}{rrrrrrrrrrrr}
8 & 20 & 11 & 3 & 0 & 0 & 1 & 0 & 0 & 2 & 3 & 2 \\
3 & 1 & 5 & 3 & 13 & 5 & 1 & 2 & 0 & 0 & 0 & 0
\end{array}
$$

$$
\begin{array}{rrrrrrrrrrrr}
6 & 26 & 0 & 0 & 3 & 0 & 0 & 0 & 0 & 0 & 3 & 3 \\
6 & 6 & 0 & 1 & 0 & 0 & 0 & 2 & 0 & 0 & 0 & 0
\end{array}
$$$$
\begin{array}{llllllllllll}
3 & 6 & 17 & 7 & 12 & 5 & 2 & 0 & 2 & 3 & 0 & 0
\end{array}
$$$$
\begin{array}{llllllllllll}
0 & 0 & 0 & 0 & 0 & 0 & 0 & 0 & 0 & 0 & 0 & 0 \\
0 & 0 & 0 & 1 & 0 & 0 & 0 & 0 & 0 & 0 & 0 & 0
\end{array}
$$$$
\begin{array}{llllllllllll}
15 & 38 & 17 & 8 & 14 & 5 & 2 & 1 & 2 & 3 & 3 & 3
\end{array}
$$$$
\begin{array}{llllllllllll}
13 & 37 & 17 & 5 & 11 & 5 & 2 & 1 & 2 & 3 & 0 & 3
\end{array}
$$$$
\begin{array}{llllllllllll}
15 & 15 & 17 & 8 & 14 & 5 & 0 & 2 & 2 & 2 & 0 * & 1
\end{array}
$$$$
\begin{array}{llllllllllll}
8 & 36 & 11 & 8 & 13 & 5 & 0 & 2 & 1 & 2 & 0 & 0
\end{array}
$$$$
\begin{array}{llllllllllll}
13 & 2 * & 16 & 6 & 14 & 5 & 0 & 1 & 0 & 0 & 0 & 0
\end{array}
$$$$
\begin{array}{llllllllllll}
15 & 22 & 16 & 8 & 14 & 5 & 2 & 2 & 2 & 2 & 0 & 0
\end{array}
$$$$
\begin{array}{llllllllllll}
4^{*} & 35 & 15 & 7 & 14 & 5 & 2 & 2 & 2 & 2 & 3 & 0
\end{array}
$$$$
\begin{array}{llllllllllll}
12 & 27 & 17 & 8 & 14 & 5 & 2 & 2 & 2 & 3 & 0 & 0
\end{array}
$$$$
\begin{array}{llllllllllll}
11 & 35 & 16 & 8 & 14 & 5 & 2 & 2 & 2 & 3 & 0 & 0
\end{array}
$$$$
\begin{array}{llllllllllll}
15 & 37 & 17 & 7 & 14 & 5 & 2 & 2 & 2 & 3 & 0 & 0
\end{array}
$$$$
\begin{array}{llllllllllll}
5 & 35 & 16 & 8 & 14 & 5 & 2 & 1 & 2 & 3 & 0 & 3
\end{array}
$$$$
\begin{array}{rlllllllllll}
15 & 38 & 17 & 7 & 13 & 4 & 2 & 2 & 2 & 3 & 3 & 3
\end{array}
$$$$
\begin{array}{llllllllllll}
14 & 38 & 17 & 8 & 14 & 5 & 2 & 2 & 2 & 3 & 3 & 3
\end{array}
$$$$
\begin{array}{llllllllllll}
14 & 38 & 17 & 8 & 14 & 5 & 2 & 2 & 2 & 3 & 3 & 3 \\
13 & 34 & 11 & 8 & 14 & 5 & 2 & 1 & 2 & 3 & 3^{*} & 1
\end{array}
$$

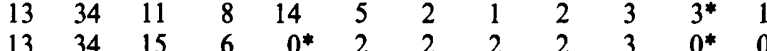$$
\begin{array}{rrrrrrrrrrrr}
13 & 34 & 15 & 6 & 0^{*} & 2 & 2 & 2 & 2 & 3 & 0^{*} & 0 \\
0 & 2 & 0 & 3 & 10^{*} & 2 & 0 & 0 & 0 & 0 & 0 & 0
\end{array}
$$ 
Table 2 (continued)

Growth $a t^{*}$ :

$10^{\circ} \mathrm{C}$

$20^{\circ} \mathrm{C}$

$45^{\circ} \mathrm{C}$

Growth in presence of $(\% w / v)^{*}$ :

$\begin{array}{rlrrrrrrrrrrrr}\text { Cluster number } & \ldots & 1 & 4 & 7 & 8 & 9 & 10 & 2 & 3 & 5 & 6 & 11 & 12 \\ \text { Number of strains } & \ldots & 15 & 38 & 17 & 8 & 14 & 5 & 2 & 2 & 2 & 3 & 3 & 3\end{array}$

Adenine (0.4)

Bismuth citrate $(0.0001)$

Crystal violet $(0.0001)$

Phenol (0.01) $(0.001)$ $(0 \cdot 1)$

Phenyl ethanol $(0.1 \%, v / v)$ $(0.2 \%, v / v)$
$(0.3 \%, v / v)$
$(0.4 \%, v / v)$

Potassium tellurite $(0.001)$ $(0.005)$ $(0.01)$

Sodium azide $(0.001)$

$$
\begin{array}{rrrrrrrrrrrr}
7 & 4 & 0 & 3 & 6 & 2 & 2 & 0 & 2 & 2 & 0 & 3 \\
15 & 10 & 17 & 8 & 13 & 5 & 2 & 2 & 2 & 2 & 0 & 3 \\
5 & 37 & 15 & 6 & 5 & 2 & 2 & 2 & 2 & 3 & 3 & 3
\end{array}
$$

$$
(0.01)
$$

Sodium chloride (3)

Teepol (0.01)

Tetrazolium (0.01)

$$
(0 \cdot 1)
$$

Thallous acetate $(0.0001)$ $(0.001)$

Growth on sole carbon source at $1 \%(w / v)^{*}$ :

$\begin{array}{rrrrrrrrrrrr}3 & 16 & 15 & 6 & 0 & 4 & 2 & 0 & 0 & 1 & 0 & 2 \\ 15 & 37 & 17 & 8 & 14 & 5 & 2 & 2 & 2 & 3 & 3 & 3 \\ 3 & 8 & 15 & 7 & 13 & 4 & 0 & 2 & 0 & 1 & 0 & 3 \\ 0 & 1 & 11 & 5 & 5 & 3 & 0 & 1 & 1 & 0 & 0 & 2 \\ 14 & 29 & 17 & 8 & 12 & 5 & 2 & 2 & 1 & 3 & 3 & 3 \\ 8 & 16 & 15 & 8 & 5 & 4 & 2 & 0 & 1 & 2 & 2 & 2 \\ 15 & 36 & 17 & 8 & 15 & 5 & 2 & 2 & 2 & 3 & 2 & 3 \\ 11 & 27 & 13 & 7 & 9 & 2 & 1 & 0 & 1 & 0 & 1 & 3 \\ 15 & 36 & 17 & 8 & 12 & 5 & 2 & 2 & 2 & 2 & 3 & 3 \\ 11 & 26 & 5 & 4 & 1 & 1 & 2 & 2 & 2 & 1 & 2 & 3 \\ 7 & 17 & 5 & 2 & 1 & 0 & 1 & 0 & 2 & 1 & 2 & 3 \\ 0 & 2 & 2 & 2 & 1 & 0 & 0 & 0 & 0 & 0 & 1 & 0 \\ 14 & 36 & 15 & 8 & 12 & 5 & 2 & 2 & 2 & 3 & 1 & 3 \\ 3 & 15 & 1 & 7 & 11 & 4 & 2 & 2 & 1 & 0 & 1 & 3 \\ 1 & 6 & 0 & 7 & 0 & 4 & 2 & 2 & 0 & 0 & 0 & 3 \\ 15 & 33 & 17 & 8 & 11 & 4 & 2 & 2 & 2 & 3 & 3 & 3 \\ 5 & 3 & 17 & 7 & 0 & 1 & 1 & 0 & 0 & 0 & 0 & 0 \\ 4 & 1 & 16 & 6 & 0 & 1 & 2 & 0 & 0 & 0 & 0 & 0 \\ 15 & 38 & 17 & 8 & 11 & 5 & 2 & 2 & 2 & 3 & 3 & 3 \\ 15 & 38 & 17 & 7 & 10 & 5 & 2 & 2 & 2 & 3 & 3 & 3 \\ 15 & 38 & 17 & 7 & 8 & 4 & 2 & 2 & 2 & 3 & 3 & 3 \\ 15 & 35 & 17 & 5 & 3 & 2 & 2 & 2 & 2 & 3 & 3 & 3 \\ 14 & 23 & 17 & 3 & 0 & 1 & 1 & 2 & 2 & 1 & 1 & 2 \\ 14 & 36 & 17 & 8 & 14 & 2 & 2 & 2 & 2 & 3 & 3 & 3 \\ 15 & 37 & 17 & 8 & 14 & 5 & 2 & 2 & 2 & 3 & 3 & 3 \\ 11 & 23 & 17 & 8 & 11 & 4 & 0 & 2 & 0 & 3 & 1 & 3 \\ 12 & 35 & 17 & 8 & 14 & 5 & 2 & 1 & 2 & 3 & 3 & 3 \\ 8 & 17 & 10 & 8 & 9 & 2 & 2 & 0 & 1 & 3 & 0 & 3 \\ 2 & 0 & 1 & 1 & 0 & 0 & 0 & 0 & 0 & 1 & 0 & 0\end{array}$

Adonitol

L(+)Arabinose

Cellobiose

Dextrin

D( - Fructose

D( - Galactose

$\mathrm{D}(+)$ Glucosamine

Glycerol

Glycogen

Lactose

Maltose

Mannitol

D(t)Mannose

L(+)Rhamnose

Starch

Sucrose

Trehalose

Xylose

At $0.1 \%(w / v)$ :

$\mathrm{D}(-)$ Alanine

L(-)Alanine

Arbutin

L(-)Proline

Protocatechuic acid

L-Serine

Sodium butyrate

$\begin{array}{rrrrrrrrrrrr}12 & 20 & 17 & 8 & 12 & 5 & 2 & 1 & 2 & 2 & 2 & 0 \\ 15 & 32 & 17 & 8 & 14 & 5 & 2 & 2 & 2 & 3 & 0 & 3 \\ 7 & 30 & 17 & 8 & 14 & 4 & 2 & 2 & 0 & 3 & 3 & 0 \\ 4 & 29 & 17 & 8 & 3 & 5 & 2 & 1 & 2 & 2 & 3 & 3 \\ 15 & 36 & 17 & 7 & 13 & 5 & 2 & 2 & 2 & 3 & 3 & 3 \\ 15 & 36 & 17 & 6 & 13 & 5 & 2 & 2 & 2 & 3 & 0 & 1 \\ 4 & 22 & 17 & 3 & 2 & 4 & 1 & 1 & 2 & 1 & 0 & 0 \\ 13 & 33 & 17 & 8 & 14 & 5 & 2 & 1 & 2 & 3 & 3 & 0 \\ 2^{*} & 34 & 16 & 8 & 14 & 5 & 2 & 2 & 2 & 1 & 0 & 0 \\ 1 & 15 & 17 & 6 & 14 & 5 & 2 & 1 & 2 & 2 & 1 & 0 \\ 13 & 35 & 17 & 0^{*} & 13 & 5 & 2 & 1 & 2 & 2 & 3 & 1 \\ 15 & 30 & 17 & 8 & 14 & 5 & 2 & 1 & 2 & 2 & 3 & 0 \\ 15 & 31 & 17 & 8 & 13 & 5 & 2 & 1 & 2 & 3 & 3 & 3 \\ 15 & 29 & 13 & 5 & 14 & 4 & 2 & 2 & 2 & 2 & 2 & 1 \\ 2 * & 32 & 17 & 2 & 13 & 5 & 2 & 1 & 2 & 3 & 3 & 3 \\ 14 & 34 & 17 & 8 & 14 & 5 & 2 & 1 & 2 & 1 & 3 & 3 \\ 3 & 29 & 17 & 8 & 9 & 4 & 2 & 1 & 2 & 2 & 3 & 3 \\ 15 & 30 & 17 & 8 & 14 & 5 & 2 & 2 & 2 & 3 & 3 & 2\end{array}$

$\begin{array}{rrrrrrrrrrrr}12 & 18 & 9 & 2 & 11 & 5 & 2 & 0 & 2 & 1 & 1 & 0 \\ 12 & 21 & 16 & 4 & 12 & 5 & 2 & 0 & 2 & 1 & 2 & 0 \\ 1 & 23 & 12 & 5 & 12 & 4 & 2 & 0 & 2 & 3 & 0 & 0 \\ 14 & 16 & 17 & 3 & 11 & 5 & 2 & 0 & 2 & 2 & 3 & 0 \\ 6 & 26 & 17 & 8 & 7 & 4 & 2 & 2 & 2 & 3 & 0 & 3 \\ 3 & 21 & 17 & 4 & 9 & 5 & 2 & 1 & 2 & 3 & 3 & 0 \\ 10 & 33 & 8 & 5 & 14 & 3 & 2 & 1 & 1 & 3 & 3 & 0\end{array}$


Table 2 (continued)

Sodium propionate

Sodium pyruvate

L-Tyrosine

Resistance to antibiotics $\left(\mu \mathrm{g} \mathrm{ml}^{-1}\right)^{*}$ :

Cephaloridine hydrochloride (2)

Demeclocycline (2)

Gentamicin sulphate (4)

Lincomycin hydrochloride (16)

Neomycin sulphate (3)

Oleandomycin sulphate (2)

Sodium benzylpenicillin (10)

Rifampicin (2)

(6)

Streptomycin sulphate (4)

Tobramycin sulphate (1)

Vancomycin hydrochloride $(0 \cdot 25)$

$\begin{array}{rrr}1 & 4 & 7 \\ 15 & 38 & 17 \\ 13 & 23 & 14 \\ 13 & 26 & 17 \\ 4 & 27 & 17 \\ & & \\ 12 & 28 & 17 \\ 5 & 3 & 16 \\ 0 & 0 & 17 \\ 0 & 0 & 15 \\ 1 & 27 & 0 \\ 0 & 0 & 0 \\ 12 & 37 & 17 \\ 3 & 32 & 17 \\ 10 & 38 & 13 \\ 1 & 26 & 2 \\ 13 & 38 & 17 \\ 2 & 30 & 17 \\ 11 & 34 & 17 \\ 5 & 25 & 17 \\ 0 & 0 & 17 \\ 0 & 0 & 17 \\ 0 & 0 & 4 \\ 0 & 0 & 0 \\ 4 & 37 & 17 \\ 0 & 16 & 2 \\ 4 & 12 & 16 \\ 0 & 0 & 1\end{array}$

$\begin{array}{rr}8 & 9 \\ 8 & 14\end{array}$

411

$6 \quad 13$

$8 \quad 10$ $\begin{array}{lllllll}10 & 2 & 3 & 5 & 6 & 11 & 12\end{array}$

$\begin{array}{lllllll}4 & 1 & 2 & 2 & 1 & 3 & 0\end{array}$ $\begin{array}{lllllll}4 & 2 & 1 & 2 & 3 & 1 & 0 \\ 5 & 2 & 0 & 2 & 3 & 2 & 0\end{array}$

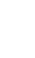

$\begin{array}{ccrrrrrrrrrrrr}\text { Cluster number } & \ldots & 1 & 4 & 7 & 8 & 9 & 10 & 2 & 3 & 5 & 6 & 11 & 12 \\ \text { Number of strains } & \ldots & 15 & 38 & 17 & 8 & 14 & 5 & 2 & 2 & 2 & 3 & 3 & 3 \\ & & 13 & 23 & 14 & 4 & 11 & 4 & 1 & 2 & 2 & 1 & 3 & 0 \\ & & 13 & 26 & 17 & 6 & 13 & 4 & 2 & 1 & 2 & 3 & 1 & 0 \\ & & 4 & 27 & 17 & 8 & 10 & 5 & 2 & 0 & 2 & 3 & 2 & 0\end{array}$

$\begin{array}{rrrllllll}8 & 9 & 5 & 0 & 0 & 0 & 3 & 3 & 1 \\ 7 & 6 & 5 & 0 & 0 & 0 & 1 & 0 & 0 \\ 6 & 0 & 4 & 0 & 0 & 0 & 0 & 3 & 0 \\ 0 & 0 & 0 & 0 & 0 & 0 & 0 & 0 & 0 \\ 4 & 0 & 3 & 0 & 0 & 0 & 0 & 3 & 0 \\ 1 & 0 & 1 & 0 & 0 & 0 & 0 & 0 & 0 \\ 8 & 10 & 5 & 2 & 0 & 0 & 3 & 3 & 0 \\ 8 & 4 & 5 & 1 & 0 & 0 & 2 & 3 & 0 \\ 8 & 14 & 5 & 2 & 1 & 2 & 0 & 3 & 3 \\ 7 & 14 & 4 & 0 & 1 & 0 & 0 & 3 & 0 \\ 8 & 12 & 5 & 2 & 1 & 2 & 3 & 3 & 2 \\ 7 & 4 & 5 & 2 & 0 & 2 & 2 & 2 & 0 \\ 8 & 7 & 5 & 0 & 0 & 0 & 3 & 3 & 1 \\ 0 & 0 & 0 & 0 & 0 & 0 & 3 & 3 & 1 \\ 8 & 0 & 5 & 0 & 0 & 0 & 1 & 0 & 1 \\ 6 & 0 & 4 & 0 & 0 & 0 & 0 & 0 & 0 \\ 5 & 0 & 5 & 0 & 0 & 0 & 1 & 0 & 0 \\ 3 & 0 & 1 & 0 & 0 & 0 & 0 & 0 & 0 \\ 5 & 1 & 5 & 2 & 0 & 2 & 2 & 3 & 3 \\ 0 & 0 & 0 & 0 & 0 & 0 & 0 & 0 & 0 \\ 8 & 6 & 5 & 1 & 0 & 0 & 0 & 2 & 1 \\ 8 & 5 & 5 & 0 & 0 & 0 & 0 & 0 & 0\end{array}$

- Tests done in Replidishes

\section{Table 3. Characteristics differentiating Saccharopolyspora species}

Key:,$+>80 \%$ of isolates positive;,$-<20 \%$ of strains positive; $v, 21-79 \%$ "isolates positive; $v(-)$, most stains negative; ND, not determined. Abbreviations: R, red; $\mathrm{O}$, orange; 1 , yellow; $\mathrm{C}$, colourless; Bf, buff; Br, brown; G, grey; P, pink; W, white.

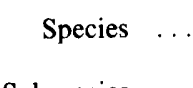

Subspecies $\ldots$

Substrate mycelium colour Aerial mycelium produced Aerial mycelium colour Soluble pigment colour Spore surface

Decomposition of:

$$
\begin{aligned}
& \text { Urea } \\
& \text { Elastin }
\end{aligned}
$$

Reduction of nitrate

Growth at:

$$
\begin{aligned}
& 10^{\circ} \mathrm{C} \\
& 45^{\circ} \mathrm{C} \\
& 50^{\circ} \mathrm{C}
\end{aligned}
$$

Utilization of:

Arabinose

Glycogen

Lactose

Rhamnose

Starch

Xylose
S. hirsuta $\dagger$

$\begin{array}{llll}\text { hirsuta } & \text { kobensis } & \text { taberi } & \\ \text { C-Bf } & \text { C-Bf-Y-P } & \text { C-Y } & \text { OY-RBr } \\ + & + & - & + \\ \text { W } & \text { W } & \text { ND } & \text { P-BrG-W } \\ \text { Y } & \text { Y-R } & \text { O-R } & \text { Y-POBr } \\ \text { Hairy } & \text { Hairy } & \text { ND } & \text { Spiny } \\ & & & \\ + & \text { ND } & - & - \\ + & + & \text { ND } & \text { ND } \\ - & + & + & + \\ & & & \\ - & - & - & - \\ + & + & \text { V } & - \\ \text { V } & - & - & - \\ & & & \\ - & - & + & + \\ \text { ND } & \text { ND } & \text { ND } & \text { ND } \\ + & \text { ND } & \text { V } & - \\ + & - & \text { ND } & \text { ND } \\ + & \text { ND } & + & + \\ + & - & \text { ND } & \text { ND }\end{array}$

S. gregorii

S. hordei

$\begin{array}{ll}\text { C-Bf } & \text { C-Bf } \\ -\bar{W} & - \\ \text { C } & \text { W } \\ \text { Smooth } & \text { C } \\ \end{array}$

$\begin{array}{ll}- & - \\ + & - \\ v(-) & v(-) \\ v & - \\ - & + \\ - & + \\ & \\ + & + \\ - & + \\ + & v \\ - & v \\ + & +\end{array}$

† Data from Lacey (1989). 


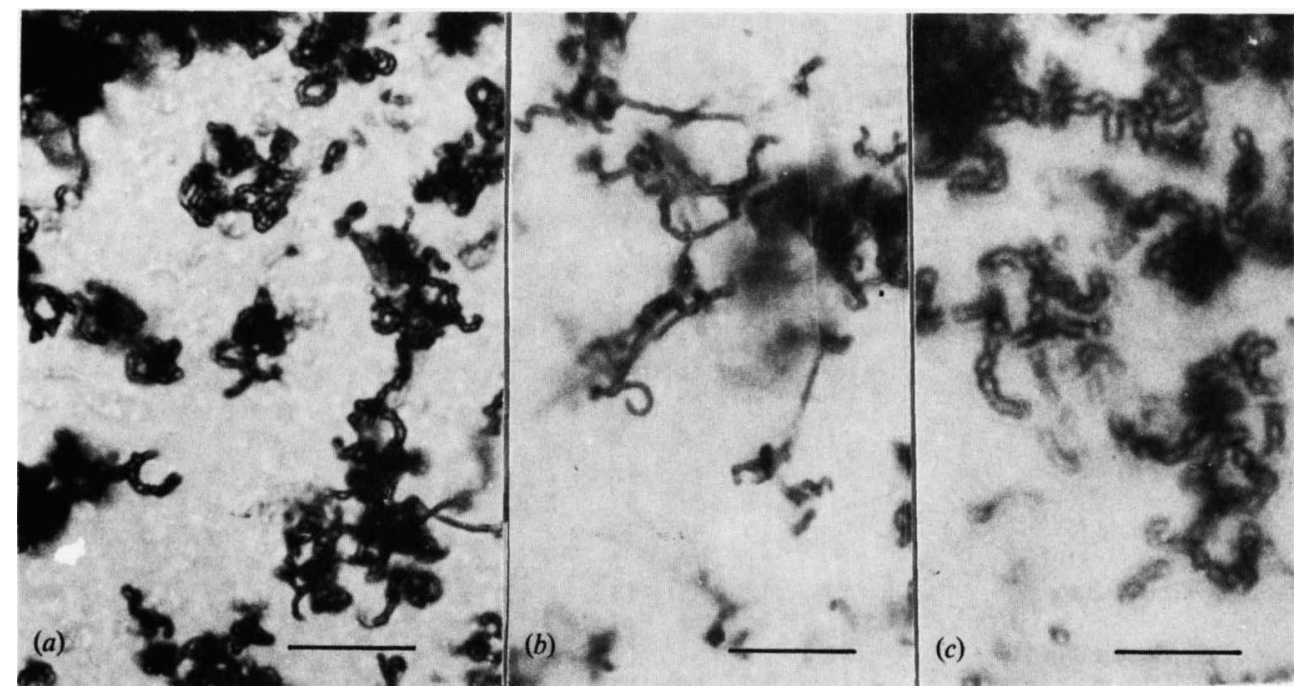

Fig. 2. Saccharopolyspora hordei. Sporulation on aerial hyphae. Bars, $20 \mu \mathrm{m}(a, b)$ and $10 \mu \mathrm{m}(c)$.

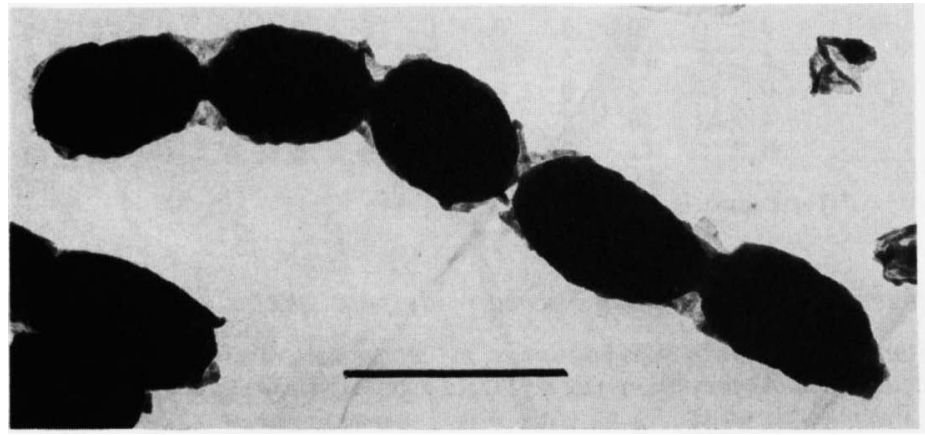

Fig. 3

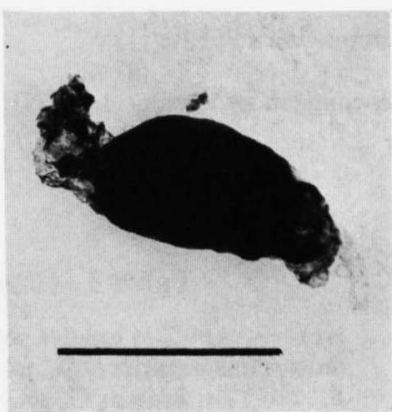

Fig. 4

Fig. 3. Saccharopolyspora hordei. Transmission electron micrograph of spore chains. Bar, $1 \mu \mathrm{m}$.

Fig. 4. Saccharopolyspora hordei. Single spore showing remnants of sheath. Bar, $1 \mu \mathrm{m}$.

Table 4. Percentage fatty acid composition of selected Saccharopolyspora strains

Assignment $\uparrow$ :

Strain

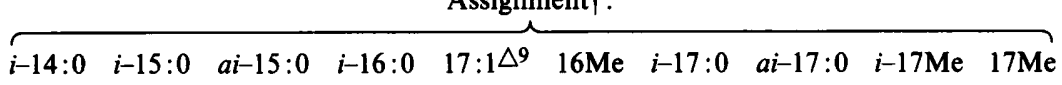

S. hirsuta

ATCC 27875*

$1 \cdot 2 \quad 16 \cdot 1$

$1 \cdot 7$

$3 \cdot 5$

$18 \cdot 1$

$14 \cdot 7$

$13 \cdot 1$

$6 \cdot 0$

S. gregorii (cluster B)

A60

$1 \cdot 7 \quad 4 \cdot 6$

$\begin{array}{lll}4.6 & 3.4 & 55.5\end{array}$

$1.4 \quad 1.2$

$1 \cdot 2 \quad 19 \cdot 0$

$\begin{array}{lll}19.0 & \text { tr } & 3.9\end{array}$

A85*

$\begin{array}{cc}2 \cdot 5 & 13 \cdot 5 \\ \operatorname{tr} & 15 \cdot 7\end{array}$

8.4

27.9

$1 \cdot 5$

$2 \cdot 0$

$5 \cdot 7 \quad 21 \cdot 1$

$5 \cdot 3 \quad 17 \cdot 7$

tr $3 \cdot 3$

$11 \cdot 3$

$33 \cdot 2$

$2 \cdot 2$

N259
Sordei (cluster A)

A54*

A65

A215

$\begin{array}{lrll}\operatorname{tr} & 5.6 & 1 \cdot 3 & 33.8 \\ \operatorname{tr} & 4.1 & 2.0 & 32.2 \\ \operatorname{tr} & 14.3 & 2.4 & 35.6\end{array}$

$\begin{array}{llll}\operatorname{tr} & 1.0 & 17.2 & 21 \cdot 1\end{array}$

$\begin{array}{llll}3.3 & \text { tr } & 14.5 & 35 \cdot 1\end{array}$

$\begin{array}{cccc}3.3 & \operatorname{tr} & 14.5 & 35.1 \\ 1.0 & 1.0 & 16.6 & 21.5\end{array}$

* Type strain.

† Trace amounts of $i-16 \triangle^{9}, 15 \mathrm{Me}, 16: 1 \Delta^{9}, 16: 0,17: 0,18 \mathrm{Me}$ and a partially characterized dimethyl branched fatty acid were also detected. Abbreviations for the FAMEs are explained by the following examples: 16:0, straight-chain hexadecanoic acid; $i$-15:0, 13-methyltetradecanoic acid; ai-15:0, 12-methyltetradecanoic acid; $i-16: 1 \triangle^{9}, 14$-methyl-cis-9,10-pentadecenoic acid; $16: 1 \triangle^{9}$, cis-9,10-hexadecenoic acid; $15 \mathrm{Me}, 10$-methylhexadecanoic acid; $i-17 \mathrm{Me}, 10,15$-dimethylhexadecanoic acid. 

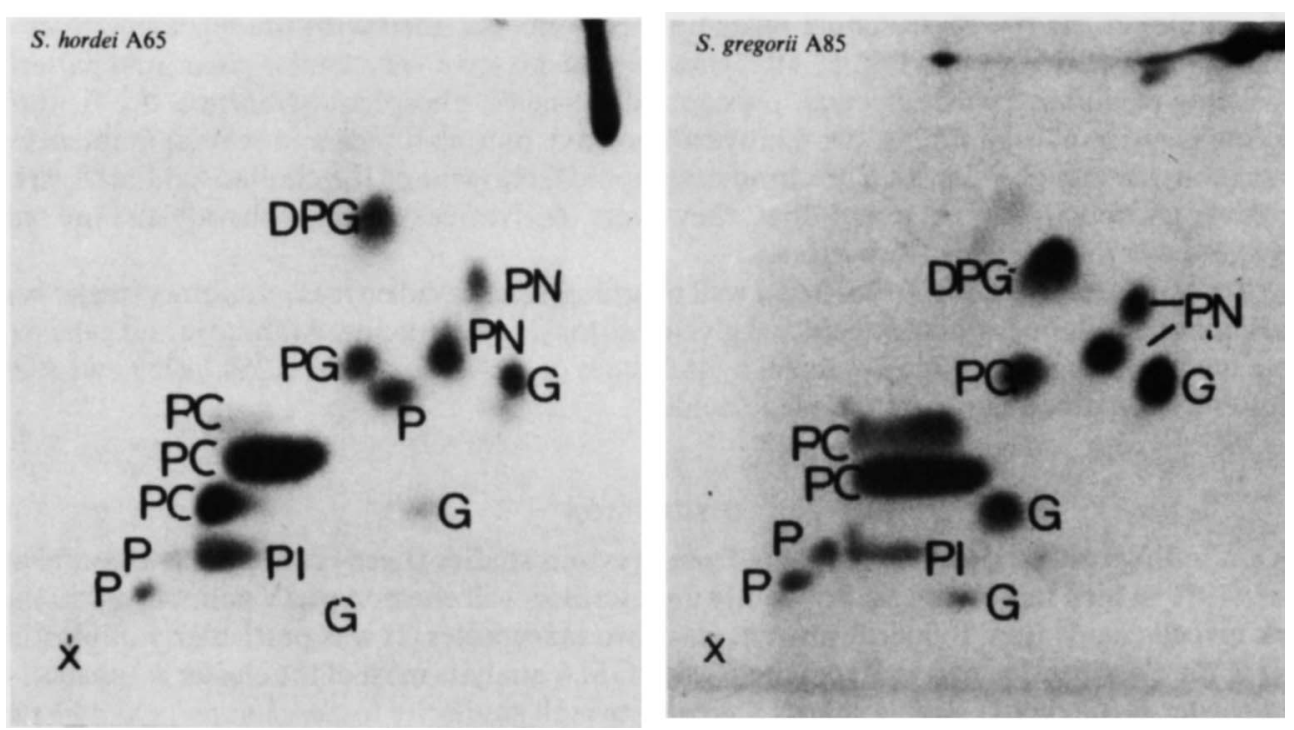

Fig. 5. Two-dimensional thin-layer chromatograms of polar lipids of Saccharopolyspora hordei A65 and Saccharopolyspora gregorii A85. DPG, diphosphatidylglycerol; G, glycolipids; P, phospholipids; PC, choline-positive phospholipids; PG, phosphatidylglycerol; PI, phosphatidylinositol; PN, ninhydrinpositive phospholipids; $\mathrm{X}$, origin.

Cluster 7 isolates differed from those assigned to other clusters in having a well-developed aerial mycelium that was white except at optimum temperatures, when it assumed a pinkish tinge (2ca, red series; Tresner \& Backus, 1963). The colony reverse was white to pale yellow. Spore chains were produced on the aerial mycelium in coils and spirals except in strain A254, where they were close to flexuous. Spores were smooth. The temperature range for growth was 20-25 to $40{ }^{\circ} \mathrm{C}$ but strain $\mathrm{A} 254$ grew up to $55^{\circ} \mathrm{C}$.

Of the single-member clusters, organism A61 was typical of the appearance of Faenia rectivirgula and grew at temperatures from 35 to $60^{\circ} \mathrm{C}$. Strain A68 resembled cluster 4 isolates. Organisms A215 and A265 resembled cluster 1 strains, but without aerial mycelium and with little fragmentation.

\section{Chemosystematic analyses}

The mean DNA base composition values derived from the two determinations performed on strains A65 (cluster 4) and A85 (cluster 1) were 72 and $74 \mathrm{~mol} \% \mathrm{G}+\mathrm{C}$, respectively.

TLC analysis of whole-organism methanolysates of the test strains of Saccharopolyspora (Table 4) revealed major components $\left(R_{F} 0.8\right)$ that corresponded to non-hydroxylated FAMEs (Minnikin et al., 1980). These strains also contained components that co-chromatographed with the methyl ester of 2-hydroxystearate $\left(R_{F} 0 \cdot 2\right)$. All the organisms tested gave qualitatively similar fatty acid profiles (Table 4) that consisted of iso-, anteiso- and 10-methyl branched saturated, iso- branched mono-unsaturated, and straight-chain saturated and mono-unsaturated fatty acids. The major components were iso-15, anteiso- 15 , iso- 16 , iso-17, anteiso- 17 and $17 \mathrm{Me}$, although the relative proportions of these compounds varied between strains. The $S$. gregorii and $S$. hordei strains contained small amounts of $i$ so- 10 -methylhexadecanoic acid $(<2 \cdot 2 \%)$ whereas $S$. hirsuta ATCC 27875 possessed a relatively large proportion of this compound $(13.1 \%)$. Mycolic acids were not detected.

Components that co-chromatographed with vitamin $\mathrm{K}_{1}$ and gave mass spectra characteristic for menaquinones were the only isoprenoid quinones found in the test strains. All isolates contained tetrahydrogenated menaquinones with nine isoprene units $\left[\mathrm{MK}-9\left(\mathrm{H}_{4}\right)\right]$ as the major isoprenologue $(60-100 \%)$ and smaller proportions of $\mathrm{MK}-8\left(\mathrm{H}_{4}\right)(0-5 \%), \mathrm{MK}-9\left(\mathrm{H}_{2}\right)(0-30 \%)$ and $\mathrm{MK}-9\left(\mathrm{H}_{6}\right)(0-13 \%)$. 
Examples of the two-dimensional polar lipid patterns obtained with the representatives of clusters 1 and 4 are shown in Fig. 5. All the test organisms gave very similar polar lipid patterns consisting of diphosphatidylglycerol, phosphatidylglycerol, phosphatidylinositol, two or three choline-positive phospholipids, two ninhydrin-positive phospholipids and several unidentified phospholipids and glycolipids. The chromatographic behaviour of the choline- and ninhydrinpositive phospholipids suggested that they were derivatives of phosphatidylcholine and phosphatidylethanolamine, respectively.

All of the cluster A and B strains had a wall peptidoglycan based on meso-diaminopimelic acid and contained alanine, glutamic acid and glycine as major amino acids. Arabinose and galactose were the major sugars. Whole-organism hydrolysates of cluster C strains A258, A259 and A261 (cluster 7) contained LL-diaminopimelic acid.

\section{DISCUSSION}

Our findings confirm and extend those from previous studies (Lacey et al., 1978; Goodfellow et al., 1979) which indicated that previously undescribed wall chemotype IV actinomycetes that lack mycolic acids from fodder formed at least two taxospecies. It was particularly interesting that in the classification derived from the $D_{\mathrm{P}}$, UPGMA analysis most of the cluster A (phenon 4) and cluster B (phenon1) strains shared a greater overall similarity to Saccharopolyspora hirsuta than to Amycolata autotrophica, Amycolatopsis orientalis and Faenia rectivirgula. Indeed, the fodder isolates and Saccharopolyspora hirsuta N745 formed a distinct aggregate group. A second, albeit chemically heterogeneous, assemblage encompassed Amycolatopsis orientalis, Saccharothrix aerocolonigenes (wall chemotype III) and cluster C (wall chemotype I) strains. The latter have properties consistent with their assignment to the genus Streptomyces (Williams et al., 1983).

Cluster composition was not markedly affected by the statistics used, or by the $4 \cdot 1 \%$ test error. However, relationships between clusters were influenced by the coefficients of association. Thus, in the $S_{\mathrm{SM}}$, UPGMA analysis, phenon 1 (cluster B) and Saccharopolyspora hirsuta N745 had a closer relationship to the Amycolatopsis, Saccharothrix and cluster C (Streptomyces sp.) strains than to fodder isolates assigned to phenon 4 (cluster A). This and earlier observations (Goodfellow et al., 1979, 1985; Athalye et al., 1985) emphasize the importance of evaluating the composition of numerically circumscribed aggregate groups using data derived from chemical and molecular systematic studies.

A combination of chemical and morphological properties are currently used to classify wall chemotype IV actinomycetes that lack mycolic acids (Goodfellow \& Minnikin, 1984; Lechevalier $e$ t al., 1986; Embley et al., 1988). The representatives of clusters A (phenon 4) and B (phenon 1) were rich in iso- and anteiso- fatty acids and contained small amounts of the diagnostic component, iso-10 methylhexadecanoic acid. These organisms also contained tetrahydrogenated menaquinones with nine isoprene units as the predominant isoprenologue and had a polar lipid pattern that included major amounts of diphosphatidylglycerol, phosphatidylglycerol, phosphatidylinositol and derivatives of phosphatidylcholine and phosphatidylethanolamine. It is evident given these chemical data that phena 1 and 4 are more closely related to Faenia and Saccharopolyspora than to the genera Actinopolyspora, Amycolata, Amycolatopsis, Pseudonocardia and Saccharomonospora (Embley et al., 1988). The chemical data recorded for Saccharopolyspora hirsuta N745 are in good agreement with previous findings (Embley et al., 1987, 1988).

The genera Faenia and Saccharopolyspora are currently distinguished by their DNA base composition (Poschner et al., 1985; Lechevalier et al., 1986; Labeda, 1987) and detailed polar lipid profiles (Embley et al., 1988). Faenia strains have DNA with relatively low $G+C$ values (66-68 mol \%), a single phosphatidylcholine component and many glycolipids; Saccharopolyspora strains have DNA richer in $\mathrm{G}+\mathrm{C}(70-77 \mathrm{~mol} \%)$, multiple phosphatidylcholine components and fewer glycolipids. On this basis the fodder isolates belonging to phena 1 and 4 have more in common with Saccharopolyspora than Faenia. Ribosomal RNA sequencing data support the association between the genus Saccharopolyspora and phenon 4 (Embley et al., 1989). 
It is evident from the chemical, morphological and numerical phenetic data that the strains assigned to phena 1 (cluster B) and 4 (cluster A) merit species status within the genus Saccharopolyspora Lacey and Goodfellow 1975. Accordingly, the species Saccharopolyspora gregorii and Saccharopolyspora hordei are proposed for phena 1 and 4, respectively. The minor phena and single-member clusters accommodating the remaining cluster A and B strains may form the nuclei of additional species, but further studies are needed to establish this.

\section{Description of Saccharopolyspora gregorii Goodfellow, Lacey, Athalye, Embley and Bowen sp. nov.}

gre.gor'i.i. ML gen.n. gregorii of Gregory; named after P. H. Gregory, a British mycologist and aerobiologist.

Colonies pale yellowish to buff with sparse white aerial mycelium at growth temperatures of about $20^{\circ} \mathrm{C}$. Substrate mycelium well developed but fragmenting extensively into coccoid elements. Sporulation or fragmentation of the aerial mycelium may sometimes be seen, forming hooks or flexuous chains. Transmission electron micrographs show mainly vegetative rods and cocci but occasionally spores with a smooth sheath. Degrades aesculin, arbutin, casein, elastin, gelatin, hypoxanthine, starch, Tweens 20 and 80, tyrosine and xanthine; utilizes adonitol, L-arabinose, fructose, galactose, glycerol, maltose, mannitol, mannose, rhamnose, sucrose, xylose, D- and L-alanine, L-proline, sodium propionate and sodium pyruvate as sole carbon sources but not glycogen, lactose, starch, trehalose or L-serine. Nitrate is not reduced and neither allantoin nor urea is hydrolysed. The organism is tolerant to a range of antibiotics and chemical inhibitors (see Table 2). Temperature range for growth: 10 to $35^{\circ} \mathrm{C}$.

The wall peptidoglycan contains alanine, glutamic acid and glycine with meso- diaminopimelic acid as the diamino acid. The principal wall sugars are arabinose and galactose. The organism is rich in iso- and anteiso- fatty acids, with 14-methylpentadecanoic and 14methylhexadecanoic acids predominating. It also contains tetrahydrogenated menaquinones with nine isoprene units as the major isoprenologue and major amounts of diphosphatidylglycerol, phosphatidylcholine, phosphatidylglycerol, phosphatidylinositol and uncharacterized phospho- and glycolipids. The mol \% G + C content of the DNA of the type strain is $74\left(T_{\mathrm{m}}\right)$.

Isolated from grass, hay, straw, barley grain and soil.

Type strain: Saccharopolyspora gregorii NCIB 12823 (A85).

\section{Description of Saccharopolyspora hordei Goodfellow, Lacey, Athalye, Embley and Bowen}

hor'.de.i. L neut. n. hordeum, barley; ML n. Hordeum, a genus of grass.

Colonies colourless to light buff, conical, rounded or wrinkled. Substrate mycelium well developed, sometimes fragmenting into coccoid elements. Aerial mycelium lacking or sparse white with short flexuous, hooked or spiral spore chains often with a beaded appearance. Transmission electron micrographs show the spores to be characteristically covered by a smooth sheath, with adjacent spores often separated by a short length of empty hypha. Degrades aesculin, arbutin, DNA, guanine, RNA, starch, testosterone, Tweens 20 and 80 , L-tyrosine and xanthine; utilizes L-arabinose, cellobiose, fructose, galactose, glycogen, glycerol, maltose, mannitol, mannose, starch, sucrose and xylose as sole carbon sources. Neither allantoin nor urea is hydrolysed. Tolerant to a range of antibiotics and chemical inhibitors (see Table 2). Temperature range for growth is $20-30{ }^{\circ} \mathrm{C}$ up to $60^{\circ} \mathrm{C}$.

The wall peptidoglycan contains alanine, glutamic acid and glycine with meso-diaminopimelic acid as the diamino acid. The principal wall sugars are arabinose and galactose. The organism is rich in iso- and anteiso- fatty acids, with 14-methylhexadecanoic acid and 14- and 15methylpentadecanoic acids predominating. It also contains tetrahydrogenated menaquinones with nine isoprene units as the major isoprenologue and major amounts of diphosphatidylglycerol, phosphatidylcholine, phosphatidylglycerol, phosphatidylinositol and uncharacterized phospho- and glycolipids. The mol \% G $+C$ content of the DNA of the type strain is $72\left(T_{\mathrm{m}}\right)$.

Isolated from barley and oat grains, grass hay, straw and sugar cane bagasse.

Type strain: Saccharopolyspora hordei NCIB 12824 (A54). 
Saccharopolyspora gregorii and Saccharopolyspora hordei can be distinguished from one another and from Saccharopolyspora hirsuta and Saccharopolyspora erythraea by biochemical, morphological, nutritional and physiological properties. Further work is needed to determine whether Saccharopolyspora species are implicated in extrinsic allergic alveolitis.

The authors are indebted to colleagues (Table 1) who kindly provided cultures. We thank Mrs Shelagh Nabb and Mrs Sheila Roberts for technical assistance. M.A. gratefully acknowledges receipt of an SERC/CASE studentship tenable at the University of Newcastle and Rothamsted Experimental Station.

\section{REFERENCES}

ATHALYE, M. (1981). Classification and isolation of actinomadurae. $\mathrm{PhD}$ thesis, University of Newcastle upon Tyne.

Athal Ye, M., Goodfellow, M., LACEy, J. \& White, R. P. (1985). Numerical classification of Actinomadura and Nocardiopsis. International Journal of Systematic Bacteriology 35, 86-98.

ColliNs, M. (1985). Isoprenoid quinone analysis in bacterial classification and identification. In Chemical Methods in Bacterial Systematics, pp. 267-287. Edited by $M$. Goodfellow \& D. E. Minnikin. London: Academic Press.

Embley, T. M., Goodfellow, M., Minnikin, D. E. \& O'DONNELL, A. G. (1984). Lipid and cell wall amino acid composition in the classification of Rothia dentocariosa. Zentralblatt für Bakteriologie, Mikrobiologie und Hygiene A257, 285-295.

Embley, T. M., Wait, R., Dobson, G. \& GoodFELLOW, M. (1987). Fatty acid composition in the classification of Saccharopolyspora hirsuta. FEMS Microbiology Letters 41, 131-135.

Embley, T. M., O’Donnell, A. G., Rostron, J. \& Goodfellow, M. (1988). Chemotaxonomy of wall type IV actinomycetes which lack mycolic acids. Journal of General Microbiology 134, 953-960.

Embley, T. M., Smida, J. \& Stackebrandt, E. (1989). The phylogeny of mycolateless wall chemotype IV actinomycetes and description of Pseudonocardiaceae fam. nov. Systematic and Applied Microbiology 11, 44-52.

Englyst, H. N. \& Cummings, J. H. (1984). Simplified method for the measurement of total non-starch polysaccharides by gas liquid chromatography of constituent sugars as alditol acetates. Analyst 109, 937-942.

GoODFEllow, M. (1986). Genus Rhodococcus Zopf 1891. In Bergey's Manual of Systematic Bacteriology, vol. 2, pp. 1472-1481. Edited by P. H. A. Sneath. Baltimore: Williams \& Wilkins.

Goodfellow, M. \& Cross, T. (1984). Classification. In The Biology of the Actinomycetes, pp. 7-164. Edited by M. Goodfellow, M. Mordarski \& S. T. Williams. London: Academic Press.

Goodfellow, M. \& Dickinson, C. H. (1985). Delineation and description of microbial populations using numerical methods. In Computer-assisted Bacterial Systematics, pp. 165-225. Edited by M. Goodfellow, D. Jones \& F. G. Priest. London: Academic Press. GoOdfellow, M. \& MINNIKIN, D. E. (1984). A critical evaluation of Nocardia and related taxa. In Biological, Biochemical, and Biomedical Aspects of Actinomycetes, pp. 583-595. Edited by L. Ortiz-Ortiz, L. F. Bojalil \& V. Yakoleff. Orlando: Academic Press.
Goodfellow, M., Alderson, G. \& LaCey, J. (1979). Numerical taxonomy of Actinomadura and related actinomycetes. Journal of General Microbiology 112 , 95-111.

Goodfellow, M., Embley, T. M. \& Austin, B. (1985). Numerical taxonomy and emended description of Renibacterium salmoninarum. Journal of General Microbiology 131, 2739-2752.

GoRDON, R. E. \& MrHM, J. M. (1962). Identification of Nocardia caviae (Erikson) comb. nov. Annals of the New York Academy of Sciences 98, 628-639.

Gregory, P. H. \& LACEY, M. E. (1963). Mycological examination of dust from mouldy hay associated with farmer's lung disease. Journal of General Microbiology 30, 75-88.

LABEDA, D. P. (1987). Transfer of the type strain of Streptomyces erythraeus (Waksman 1923) Waksman and Henrici 1948 to the genus Saccharopolyspora Lacey and Goodfellow 1975 as Saccharopolyspora erythraea sp. nov. and description of a neotype strain for Streptomyces erythraeus. International Journal of Sytematic Bacteriology 37, 19-22.

LACEY, J. (1971). The microbiology of moist barley storage in unsealed silos. Annals of Applied Biology 69, 187-212.

LACEY, J. (1981). Airborne actinomycete spores as respiratory allergens. Zentralblatt für Bakteriologie, Mikrobiologie und Hygiene (Abteilung I), Supplement $11,243-250$.

LACEY, J. (1988). Actinomycetes as biodeteriogens and pollutants of the environment. In Actinomycetes in Biotechnology, pp. 359-432. Edited by M. Goodfellow, S. T. Williams \& M. Mordarski. London: Academic Press.

LACEY, J. (1989). Genus Saccharopolyspora Lacey and Goodfellow 1975, $77^{\mathrm{AL}}$. In Bergey's Manual of Systematic Bacteriology, vol. 4, pp. 2382-2386. Edited by S. T. Williams, M. E. Sharpe \& J. Holt. Baltimore: Williams \& Wilkins.

LACEY, J. \& GoOdFEllow, M. (1975). A novel actinomycete from sugar cane bagasse: Saccharopolyspora hirsuta gen. et sp. nov. Journal of General Microbiology 88, 75-85.

LACEY, J., GoOdFellow, M. \& Alderson, G. (1978). The genus Actinomadura Lechevalier and Lechevalier. Zentralblatt für Bakteriologie, Parasitenkunde, Infektionskrankheiten und Hygiene (Abteilung I), Supplement 6, 107-117.

Lechevalier, M. P. \& LechevalieR, H. A. (1970). Chemical composition as a criterion in the classification of aerobic actinomycetes. International Journal of Systematic Bacteriology 20, 435-444.

Lechevalier, M. P., Prauser, H., Labeda, D. P. \& 
RUAN, J.-S. (1986). Two new genera of nocardioform actinomycetes: Amycolata gen. nov. and Amycolatopsis gen. nov. International Journal of Systematic Bacteriology 36, 29-37.

MARMUR, J. (1961). A procedure for the isolation of deoxyribonucleic acid from microorganisms. Journal of Molecular Biology 3, 208-218.

Minnikin, D. E., Hutchinson, I. G., Caldicott, A. B. \& Goodfellow, M. (1980). Thin-layer chromatography of methanolysates of mycolic acid containing bacteria. Journal of Chromatography 188, 221-233.

MinNikin, D. E., O'DonNell, A. G., Goodfellow, M., Alderson, G., Athalye, M., SchaAl, A. \& ParletT, J. H. (1984). An integrated procedure for the extraction of isoprenoid quinones and polar lipids. Journal of Microbiological Methods 2, 233-241.

O'Donnell, A. G., Goodfellow, M. \& MinNikin, D. E. $(1982 a)$. Lipids in the classification of Nocardioides: reclassification of Arthrobacter simplex (Jensen) Lochhead in the genus Nocardioides (Prauser) emend. O'Donnell et al. as Nocardioides simplex comb. nov. Archives of Microbiology 133, 323-329.

O'Donnell, A. G., Goodfellow, M. \& Minnikin, D. E. $(1982 b)$. The analysis of actinomycete wall amino acids by gas chromatography. FEMS Microbiology Letters 15, 75E-78E.

Poschner, J., KROPPENSTEDT, R. M., FisCher, A. \& STACKEBRANDT, E. (1985). DNA :DNA reassociation and chemotaxonomic studies in Actinomadura, Microbispora, Microtetraspora, Micropolyspora and Nocardiopsis. Systematic and Applied Microbiology 6, 264-270.
SNEATH, P. H. A. (1968). Vigour and pattern in taxonomy. Journal of General Microbiology 54, 1-11.

SNEATH, P. H. A. \& SoKal, R. R. (1973). Numerical Taxonomy. The Principles and Practice of Numerical Taxonomy. San Francisco: W. H. Freeman.

Sneath, P. H. A. \& Stevens, M. (1967). A divided Petri dish for use with multipoint inoculators. Journal of Applied Bacteriology 30, 495-497.

SOKAL, R. R. \& MiChenER, C. D. (1958). A statistical method for evaluating systematic relationships. Kansas University Science Bulletin 38, 1409-1438.

StanecK, J. L. \& RoberTs, G. D. (1974). Simplified approach to identification of aerobic actinomycetes by thin-layer chromatography. Applied Microbiology 28, 226-231.

Tresner, H. D. \& BaCkus, E. J. (1963). System of colour wheels for streptomycete taxonomy. Journal of Applied Microbiology 11, 335-338.

WAGNER, H., HoRHAMmer, L. \& WolfF, L. (1961). Dunnschicht-chromatographie von Phosphatiden und Glycolipiden. Biochemische Zeitschrift 334, 175-184.

Williams, S. T., Goodfellow, M., Alderson, G., Wellington, E. M. H., Sneath, P. H. A. \& Sackin, M. J. (1983). Numerical classification of Streptomyces and related genera. Journal of General Microbiology 129, 1743-1813.

Williams, S. T., Sharpe, M. E. \& Holt, J. (editors) (1989). Bergey's Manual of Systematic Bacteriology, vol. 4. Baltimore: Williams \& Wilkins. 